\title{
Depletion of pulmonary intravascular macrophages partially inhibits lipopolysaccharide-induced lung inflammation in horses
}

\author{
Om P. PARBHAKAR ${ }^{\mathrm{a}}$, Tanya DuKE ${ }^{\mathrm{b}}$, Hugh G.G. TOWNSEND ${ }^{\mathrm{c}}$, \\ Baljit SINGH ${ }^{\mathrm{a}, \mathrm{d} *}$ \\ ${ }^{a}$ Department of Veterinary Biomedical Sciences, University of Saskatchewan, 52 Campus Drive, \\ Saskatoon, SK S7N 5B4, Canada \\ b Small Animal Clinical Sciences, University of Saskatchewan, 52 Campus Drive, Saskatoon, \\ SK S7N 5B4, Canada \\ ${ }^{c}$ Large Animal Clinical Sciences, University of Saskatchewan, 52 Campus Drive, Saskatoon, \\ SK S7N 5B4, Canada \\ d Immunology Research Group and Departments of the University of Saskatchewan, 52 Campus Drive, \\ Saskatoon, SK S7N 5B4, Canada
}

(Received 16 April 2004; accepted 25 November 2004)

\begin{abstract}
Horses are unique in their extreme sensitivity to endotoxin-induced cardio-pulmonary shock and mortality. The mechanisms behind increased sensitivity of the horse to endotoxin remain unknown. Pulmonary intravascular macrophages (PIMs) are pro-inflammatory cells occurring in horses. Because the functions of equine PIMs in endotoxemia remain unknown, we studied the role played by equine PIMs in endotoxin-induced pulmonary pathophysiology. We achieved this by using a recently developed protocol to deplete PIMs in order to compare lipopolysaccharide (LPS)induced pulmonary responses in horses with or without PIMs. Horses treated with gadolinium chloride (GC; $10 \mathrm{mg} / \mathrm{kg}$ intravenous) to deplete PIMs or endotoxin-free saline $(n=4)$ were injected with Escherichia coli LPS (E. coli LPS; $50 \mathrm{ng} / \mathrm{kg}$ intravenously) $48 \mathrm{~h}$ after GC or saline. Control horses $(n=5)$ received two injections of endotoxin-free saline at $48 \mathrm{~h}$ intervals. All the horses were euthanized $2 \mathrm{~h}$ after LPS or saline challenge. Immunohistology for the PIMs showed their reduced numbers in GC-treated horses. The LPS treatment of normal and GC-treated horses increased diastolic and systolic pulmonary arterial pressures at 30 min compared to the saline-treated horses $(P<0.05)$. However, horses pre-treated with GC did not have an LPS-induced increase in mean pulmonary arterial pressure compared to the LPS-treated horses $(P<0.05)$. Light and electron microscopic immunocytochemistry detected extensive labeling for LPS in PIMs of LPS-treated horses. Both the LPS-treated groups had more alveolar septal cells positive for TNF- $\alpha$ and IL-1 $\beta$ compared to control horses, which did not receive LPS $(P<0.05)$. However, GC-treated horses challenged with the LPS showed less IL-1 $\beta$-positive cells $(P<0.05)$. Immuno-electron microscopy localized TNF- $\alpha$ and IL-1 $\beta$ in PIMs. These new data show that PIMs endocytose LPS and contain TNF- $\alpha$ and IL-1 $\beta$ and their depletion partially inhibits LPS-induced pulmonary inflammatory responses.
\end{abstract}

PIMs / TNF- $\alpha$ / IL-1 $\beta$ / gadolinium chloride / light and electron microscopic immunocytochemistry

* Corresponding author: baljit.singh@usask.ca 


\section{INTRODUCTION}

Horses are unique in their extreme sensitivity to endotoxin-induced cardio-pulmonary shock and mortality; however, the mechanisms of these phenomena remain largely unknown [21, 31]. The mortality associated with endotoxemia causes significant economic damage to the equine industry [21,31]. Therefore, it is of significance to understand the mechanisms that make the horse more sensitive to endotoxin-induced cardio-pulmonary pathophysiology in order to better identify the therapeutic targets.

Endotoxemia is characterized by global activation of inflammatory cells, secretion of pro-inflammatory mediators and development of shock and multiple organ failure $[11,18]$. Endotoxins are highly toxic lipopolysaccharides (LPS) that are released during rapid growth or death of Gram negative bacteria. Monocytes and macrophages use their CD14 and Toll-like receptor-4 to bind and endocytose LPS, and to initiate cell signaling [1]. The internalized LPS escapes from endosomes, interacts with mitochondria and Golgi complexes, and enters the nuclei of monocytes and macrophages to activate proinflammatory gene transcription [12, 22, 35]. Therefore, macrophages play a central role in the host response to endotoxins.

Pulmonary intravascular macrophages (PIMs) are relatively newly identified mononuclear phagocytes that occur in domestic animal species such as cattle, horses, sheep, pigs and cats $[3,4,16,32,33]$. The species that contain PIMs show marked pulmonary hypertension following infusion of tracer particles and are highly susceptible to acute lung diseases [19, 28-30]. Neonatal lambs that normally have very few PIMs show little or no pulmonary hypertension following intravenous infusion of tracers or endotoxins. But pulmonary hypertension is observed in two-week old lambs that have sequestered PIMs. This hypertensive response, however, is abolished following removal of PIMs [27]. In vitro evidence shows that porcine PIMs can metabolise arachidonic acid
$[7,8]$. We have recently shown that bovine PIMs contain TNF- $\alpha$ and their depletion significantly inhibits the accumulation of inflammatory cells and pathology in acute lung disease in calves [26]. These data suggest a pro-inflammatory role for PIMs in acute lung diseases in the host species.

We have reported that horses contain PIMs, which are activated following phagocytosis of vascular tracers and exposure to halothane $[4,5,25]$. Longworth et al. reported physiological evidence that endotoxin-induced pulmonary arterial hypertension is neutralized following treatment with a detergent; an effect they speculated was due to the blockade of PIMs [17]. Currently, specific contributions of equine PIMs to endotoxin-induced pulmonary responses remain largely unknown. This prompted us to examine the in vivo roles of PIMs in LPSinduced early pulmonary vascular responses and lung inflammation. Our data show that equine PIMs endocytose intravenously infused LPS and contain pro-inflammatory cytokines. Their depletion inhibits LPSinduced accumulation of inflammatory cells.

\section{MATERIALS AND METHODS}

\subsection{Animal groups}

The experimental protocols for this study were approved by the Animal Care Committee of the University of Saskatchewan in accordance with the national guidelines for the use of animals in research. Horses ( $n=13) 1-2$ years of age were purchased from the Saskatoon Auction Market. These horses were acclimatized to new housing environment for a week and were given regular physical examinations.

\subsection{Pressure measurements}

The procedure used for the measurement of pulmonary pressures has been described previously [23]. Briefly, the right jugular vein of all horses was aseptically catheterized with an 8 Fr catheter introducer sheath 
following sedation with xylazine $(0.5 \mathrm{mg} / \mathrm{kg}$ iv; Rompun, Bayer Inc. Etobicoke, Canada). Following this, a thermistor tipped triple lumen pulmonary artery balloon catheter (Edwards Swan-Ganz Catheter, Baxter Corporation, Toronto, Canada) was positioned so that the distal end was in the pulmonary artery, and secured. All intravenous catheters were flushed with heparinized saline solution to keep them patent. A silicon chip strain gauge transducer (Truwave Disposable Transducer, Baxter Corporation, Toronto, Canada), previously calibrated with a mercury manometer, was zeroed to the level of the right atrium. This was used to measure systolic, diastolic and mean pulmonary arterial pressures through the distal port of the pulmonary artery catheter. The pressures were displayed on a physiological monometer (Propaq 104; Protocol Systems Inc., Beaverton, USA).

\subsection{Treatments}

Four horses were administered $500 \mathrm{~mL}$ of endotoxin-free physiological saline intravenously while four horses were administered GC (10 mg/kg body weight intravenously; Sigma Co, St. Louis, USA) in $500 \mathrm{~mL}$ of physiological saline solution over $20 \mathrm{~min}$ through a sideport of the introducer sheath. Pulmonary arterial pressures were recorded three times: immediately prior to the start of infusion (0 $\mathrm{min})$, after $50 \%(10 \mathrm{~min})$ and after $100 \%$ (20 min) of the $500 \mathrm{~mL}$ infusion. Forty-eight hours after the completion of these treatments the horses were administered $E$. coli LPS (50 ng/kg body weight; B0128:12; Sigma Co, St. Louis, USA) in physiological saline over $20 \mathrm{~min}$. Control horses $(n=5)$ were administered equal volumes of the endotoxin-free saline twice $48 \mathrm{~h}$ apart. Pulmonary arterial pressures were recorded before the treatment and at 15 min intervals up to $2 \mathrm{~h}$ after the LPS or saline treatments, when the horses were euthanized (Euthenol; $10 \mathrm{~mL} / 50 \mathrm{~kg}$ body weight intravenously; Biomeda-MTS Animal Health, Mississauga, Canada).

\subsection{Tissue fixation}

Horses were euthanized and tissues collected as described previously [23]. Briefly, the lungs were fixed in situ for $30 \mathrm{~min}$ by pouring $5 \mathrm{~L}$ of $2 \%$ paraformaldehyde and $0.5 \%$ glutaraldehyde in $0.1 \mathrm{M}$ sodium cacodylate buffer ( $\mathrm{pH}$ 7.2) into the trachea. The lungs were removed en bloc from 7 predetermined areas of each lung and fixed in $4 \%$ paraformaldehyde for $24 \mathrm{~h}$ and embedded in paraffin. For immuno-electron microscopy, lung pieces of approximately $1 \mathrm{~mm}^{3}$ were fixed in $2 \%$ paraformaldehyde and $0.1 \%$ glutaraldehyde in $0.1 \mathrm{M}$ sodium cacodylate buffer ( $\mathrm{pH}$ 7.2) for $3 \mathrm{~h}$. These pieces were dehydrated and embedded in LR-White (Electron Microscopy Sciences, Hatfield, USA).

\subsection{Light microscopy}

\subsubsection{Histopathology}

Sections 5-7 $\mu \mathrm{m}$ thick were prepared from all the blocks from each of the horses. These were stained with hematoxylin-eosin.

\subsubsection{Immunohistology with E. coli LPS, TNF- $\alpha$ and IL-1 $\beta$ antibodies}

The immunohistology protocols have been described previously [23]. Briefly, sections from paraffin blocks were dewaxed, rehydrated and treated with pepsin (2 $\mathrm{mg} / \mathrm{mL} 0.01 \mathrm{~N} \mathrm{HCl}$; Sigma Co.) for 90 min to unmask the antigen sites. Following incubation in $0.5 \%$ hydrogen peroxide for $20 \mathrm{~min}$ and blocking with $1 \%$ bovine serum albumin for $30 \mathrm{~min}$, the sections were exposed to primary antibodies against TNF- $\alpha$ (1:75; Serotec Ltd. Raleigh, USA) IL-1 $\beta$ (1:75; Serotec Ltd. Raleigh, USA) and E. coli LPS (1:25; B0128:12; Calbiochem Inc. La Jolla, USA) for $2 \mathrm{~h}$ and appropriate horseradish peroxidase-conjugated secondary antibodies (DAKO A/S Mississauga, Canada). The color was developed with a commercial kit (Vector Laboratories, Burlington, Canada). Selected lung sections from each group were stained with 


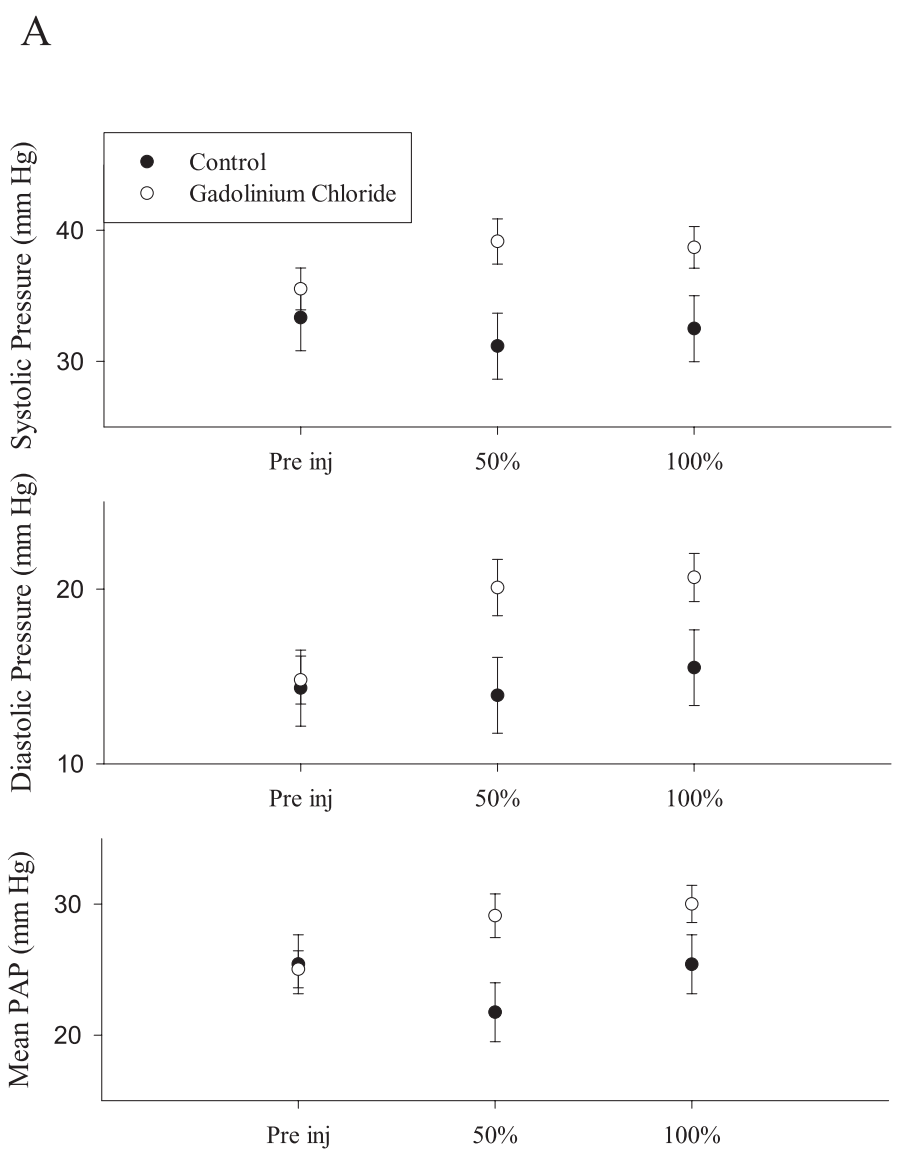

Figure 1. There were no differences in systolic, diastolic and mean pulmonary arterial pressures (A) between saline-treated $(\bullet)$ and GC-treated horses $(O)$ before and after the treatment. Systolic and diastolic pressures were significantly higher in both the LPS ( $\boldsymbol{\nabla}$ and $\bigcirc)$ treated groups (B) at 30 min compared to the saline-treated controls $(\bullet)(* * P<0.05)$. There were no differences in mean pulmonary arterial pressure between the GC + LPS group $(\boldsymbol{\nabla})$ compared to the controls $(\bullet)$. However, mean pulmonary arterial pressure was significantly less $(* P<0.05)$ in the GC + LPS horses $(\nabla)$ compared to saline + LPS horses $(O)$.

MAC-387 (1:75; Serotec Ltd. Raleigh, USA), which we had previously used to identify equine PIMs, in order to assess depletion of PIMs [23]. Immunohistochemical controls included incubation with isotypematched immunoglobulins, staining of lung vasculature with anti-von Willebrand Factor antibody and omission of primary or both primary and secondary antibodies.

\subsubsection{Quantification of the cells}

Cells stained with TNF- $\alpha$ and IL- $1 \beta$ antibodies were counted over 20 fields under oil immersion $(\times 100)$ from one section from each tissue block (7 blocks/lung; 14 blocks/horse). Each field comprised $0.025 \mathrm{~mm}^{2}$ of tissue area, which resulted in $0.50 \mathrm{~mm}^{2} /$ section and $7.0 \mathrm{~mm}^{2} /$ horse. The 


\section{B}
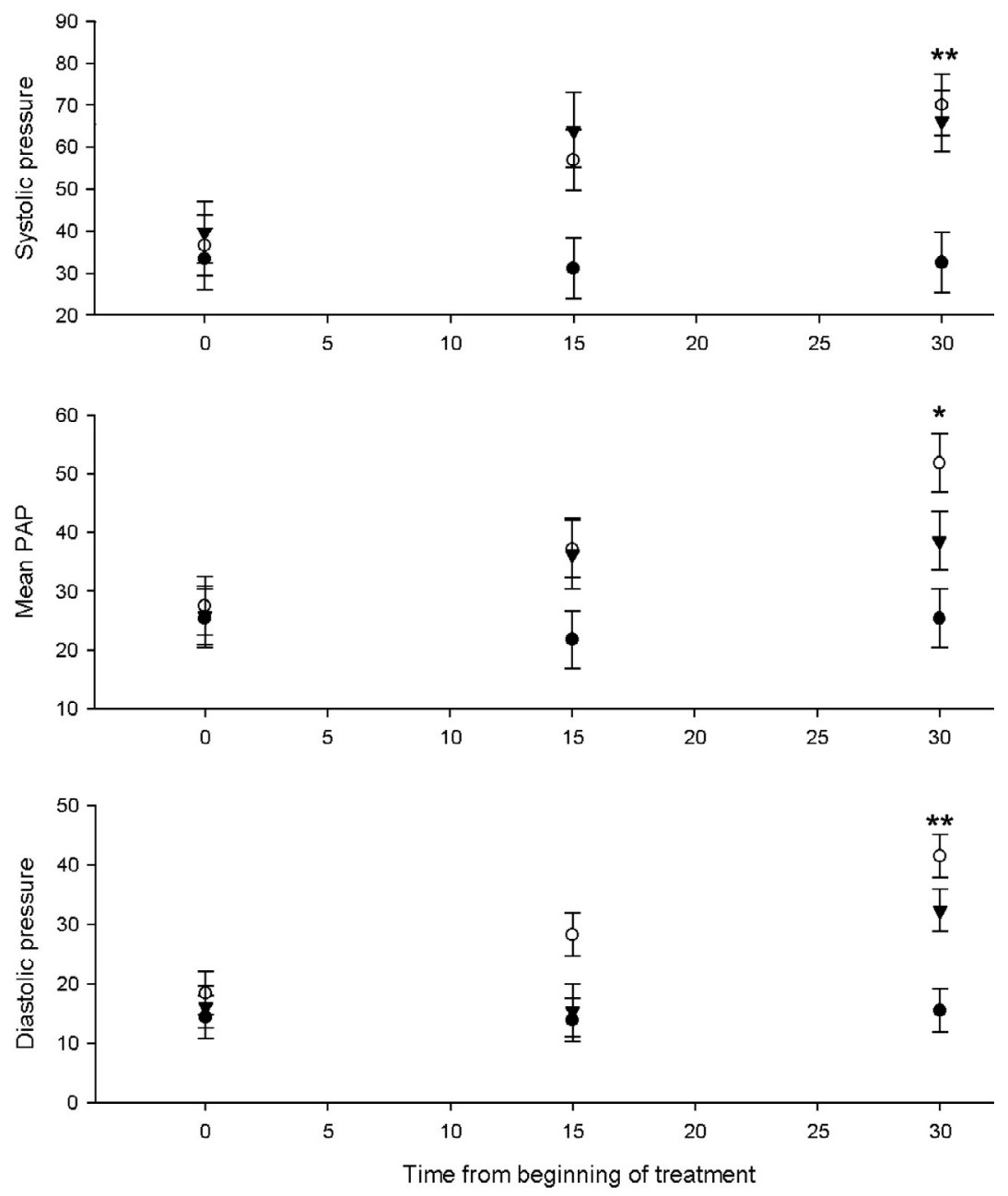

Figure 1. Continued.

slides were coded and the evaluator was blinded to the treatment groups.

\subsection{Immuno-electron microscopy}

We selected a few tissue blocks from each horse for immuno-electron microscopy to confirm staining with antibodies. Thin sections $(100 \mathrm{~nm})$ were placed on nickel grids and blocked with $1 \%$ normal goat serum followed by incubation with primary antibodies against TNF- $\alpha$ (1:75) or IL- $1 \beta$ $(1: 75)$ or the LPS $(1: 25)$ and $10 \mathrm{~nm}$-gold conjugated goat anti-mouse secondary antibody (1:100; Biocell International, Rancho Dominguez, USA). The sections were stained with uranyl acetate and lead citrate. The controls for the immuno-electron microscopy included omission of primary antibody or 
replacing primary antibody with an isotype-matched immunoglobulin.

\subsection{Statistical analyses}

Differences in numbers of cells positive for TNF- $\alpha$ and IL- $1 \beta$ between horses treated with the LPS, LPS and GC, or saline alone were analyzed by first ranking the data and then performing a one-way ANOVA on the ranks. The means of the ranks were separated using the Tukey test. The pressure readings among the treatment groups were compared by performing a non-parametric ANOVA with repeated measures over time. In those instances where the ANOVA were significant, differences among the groups at individual time points were examined using the Wilcoxon Rank Sum test. Differences for all analyses were considered significant when $P \leq 0.05$.

\section{RESULTS}

\subsection{Physiological responses to LPS infusion}

GC alone did not cause changes in systolic, diastolic or mean pulmonary arterial pressures (Fig. 1A). Both the LPS-treated groups showed increases in diastolic and systolic pulmonary arterial pressures at $30 \mathrm{~min}$ compared to the saline-treated horses $(P<0.05$; Fig. 1B) followed by a return to the baseline by $120 \mathrm{~min}$ post-treatment (data not shown). Those horses given saline before LPS demonstrated increased mean pulmonary arterial pressures compared to the controls at $30 \mathrm{~min}$ after the LPS treatment. However, the horses pre-treated with GC did not show LPS-induced increase in mean pulmonary arterial pressure compared to the saline-treated horses $(P<0.05)$ (Fig. 1B).

\subsection{Histopathology}

Staining with H\&E showed only mild congestion in the lungs of LPS-treated horses compared to the control and those given GC before the LPS (Figs. 2A-2C).

\subsection{PIMs endocytose $E$. coli LPS}

Because there was no evidence of localization of the LPS in the lungs of LPStreated horses, we first performed immunostaining on lung sections with an antiLPS antibody. Sections from the lungs of horses incubated with only secondary antibody (Fig. 3A) or isotype-matched antibody (data not shown) lacked staining while antivWF antibody delineated lung vasculature (Fig. 3B). Staining for E. coli LPS was observed in large discrete cells in alveolar septa in the LPS-treated but not in salinetreated horses (Figs. 3C and 3D). Immunogold electron microscopy showed intense gold labeling for LPS in the cytosol and nuclei of PIMs of the LPS-treated horses (Fig. 3E). The lung microvascular endothelium and alveolar epithelium were also positive for LPS (Fig. 3E). Immuno-gold electron microscopy controls showed vWF staining in the Weibel-Palade bodies of the endothelial cells (Fig. 3F).

\subsection{PIMs contain IL-1 $\beta$ and TNF- $\alpha$}

Lung sections from the saline-treated horses contained only an occasional septal cell stained for IL-1 $\beta$ (Fig. 4A) or TNF- $\alpha$ (Fig. 5A). Lung sections from horses treated with LPS showed IL-1 $\beta$ (Fig. 4B) or TNF- $\alpha$ (Fig. 5B) staining in many large cells in the alveolar septa. In addition, the IL- $1 \beta$ antibody caused diffuse staining in the alveolar septa in lung sections from the LPS-treated horses (Fig. 4B). Immuno-gold electron microscopy detected IL- $1 \beta$ and TNF- $\alpha$ in PIMs of horses treated with LPS (Figs. 4C and 5C).

\subsection{Quantification of cells positive for IL-1 $\beta$ and TNF- $\alpha$}

First, we performed staining with MAC387 antibody to again determine depletion of PIMs in GC-treated horses. The data show a reduction in PIM numbers in GCtreated horses compared to the controls (Figs. 6A and 6B) as demonstrated previously [23]. In order to determine the effects 


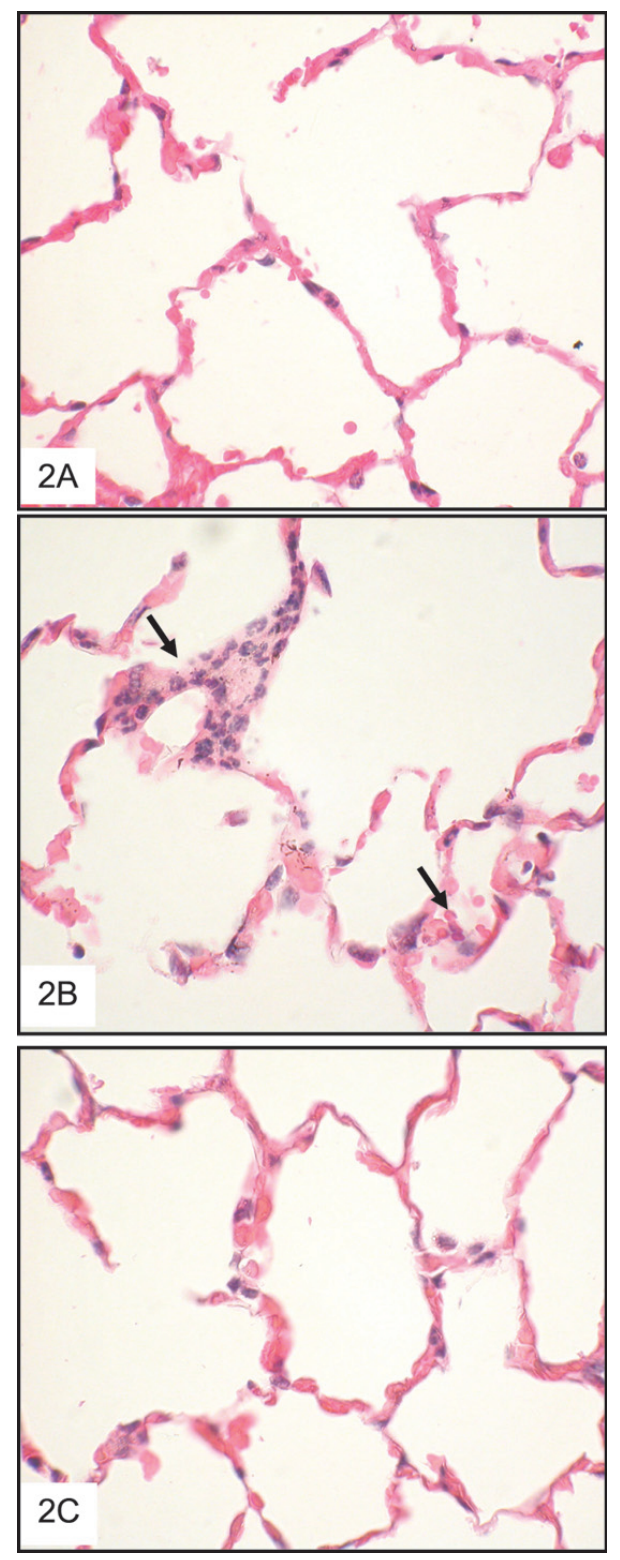

Figure 2. Lung sections from control (A) and GC + LPS horses (C) show normal lung histology. Lung sections from saline + LPS horses (B) show mild congestion in the alveolar septa (arrows). A-C: $\times 200$ (for a colour version of this figure see www.edpsciences.org/vetres).

of PIM depletion on the LPS-induced accumulation of cytokine-positive cells, we counted the numbers of cells stained for TNF- $\alpha$ and IL- $\beta$. Control horses administered LPS showed an increase in cells positive for TNF- $\alpha$ or IL- $1 \beta$ compared to those treated with endotoxin-free saline $(P<$ 0.05; Fig. 6C). PIM-depleted horses treated with LPS showed reduced numbers of cells positive for IL- $1 \beta$ but not for TNF- $\alpha$, compared to LPS-treated control horses $(P<$ $0.05)$.

\section{DISCUSSION}

It has been known that horses are extremely sensitive to endotoxin-induced cardio-pulmonary shock and mortality [21]. The cellular and molecular mechanisms of this sensitivity, however, are still a mystery. We examined the PIMs as the central phagocytic and pro-inflammatory cell in the LPS-induced pulmonary vascular and inflammatory responses. The data show that equine PIMs endocytose blood-borne E. coli LPS and contain TNF- $\alpha$ and IL- $1 \beta$. Our data further show that PIM depletion reduces the LPS-induced elevations in mean pulmonary arterial pressure and the increase in lung cells positive for IL-1 $\beta$. These data are the first to directly implicate PIMs as one of the critical pro-inflammatory cells in the LPS-induced pulmonary responses in the horses.

First, we determined if PIMs endocytose vascular E. coli LPS. Equine PIMs revealed intense labeling for LPS in their cytoplasm and nuclei to suggest its uptake from circulation. LPS was also detected in capillary endothlelial and alveolar epithelial cells to suggest translocation of LPS across the blood-air barrier. Such localization of LPS has been reported in sheep [24]. There is previous direct and indirect evidence of rapid and preferential removal of bloodborne endotoxins by PIMs in sheep [9, 24, 34]. Immuno-gold electron microscopy localized LPS in the endosomes, cytoplasm and nuclei of sheep PIMs within 10 min of the intravenous treatment [24]. Therefore, our data confirming LPS in equine PIMs 

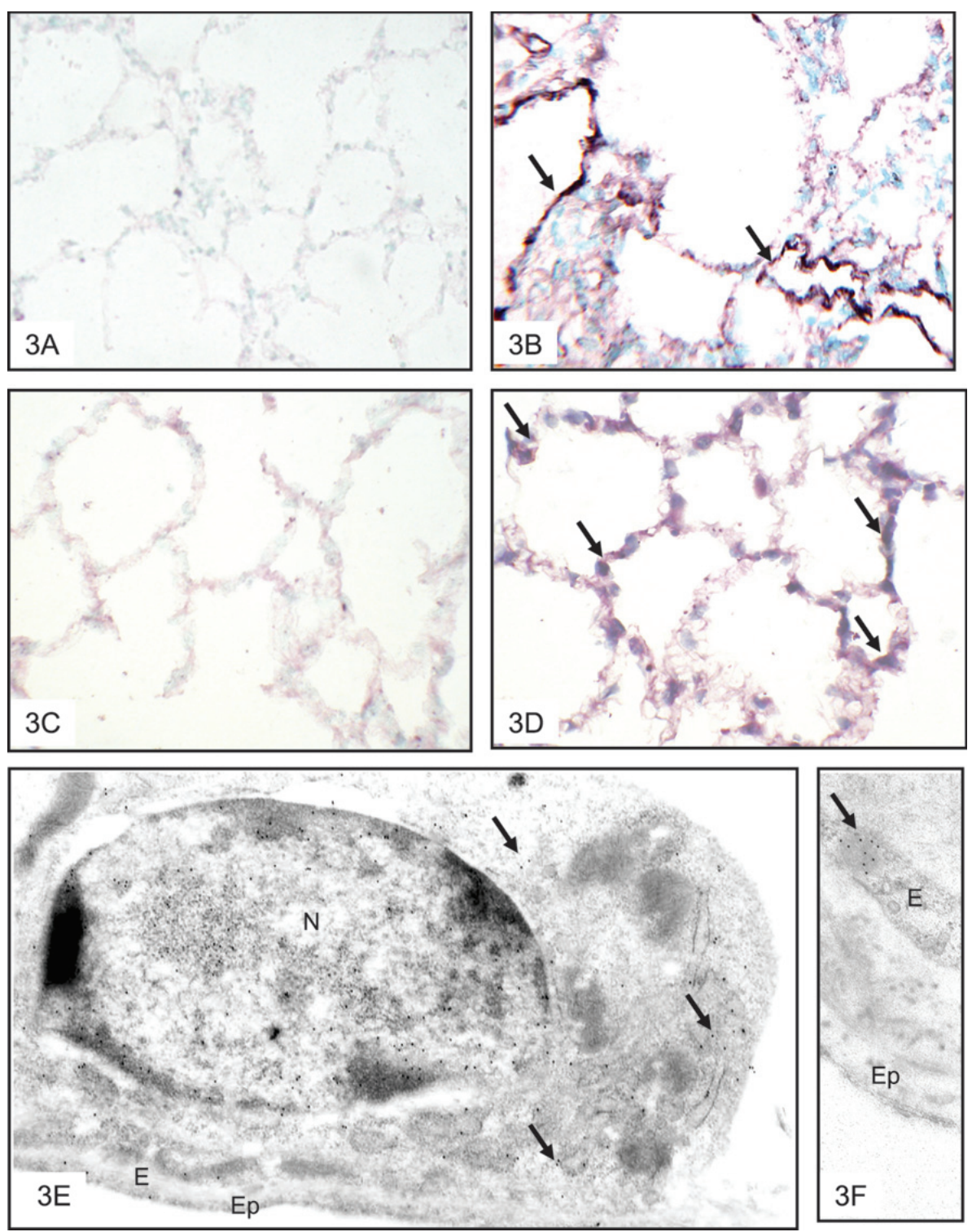

Figure 3. E. coli immunocytochemistry on lung sections from saline + LPS horses: Lung sections from a saline-treated control horse incubated with only secondary antibody (A) showed no staining while vWF antibody (B) delineated the lung vasculature (arrows). E. coli LPS antibody did not stain lung sections from the saline-treated control horses $(\mathrm{C})$ while intense staining is seen in the alveolar septa and large septal cells (arrows) of saline + LPS-treated horses (D). An immuno-gold electron micrograph (E) shows intense labeling with gold particles (arrows) of a PIM in a lung section from a saline + LPS-treated horse. The alveolar epithelial cell (Ep) and endothelial cell (E) show labelling for the LPS. Weibel-Palade body (arrow) in a lung capillary endothelial cell (E) show staining for the von-Willebrand Factor (F). A-D: $\times 400$; E-F: $\times 37500$ (for a colour version of this figure see www.edpsciences.org/vetres). 


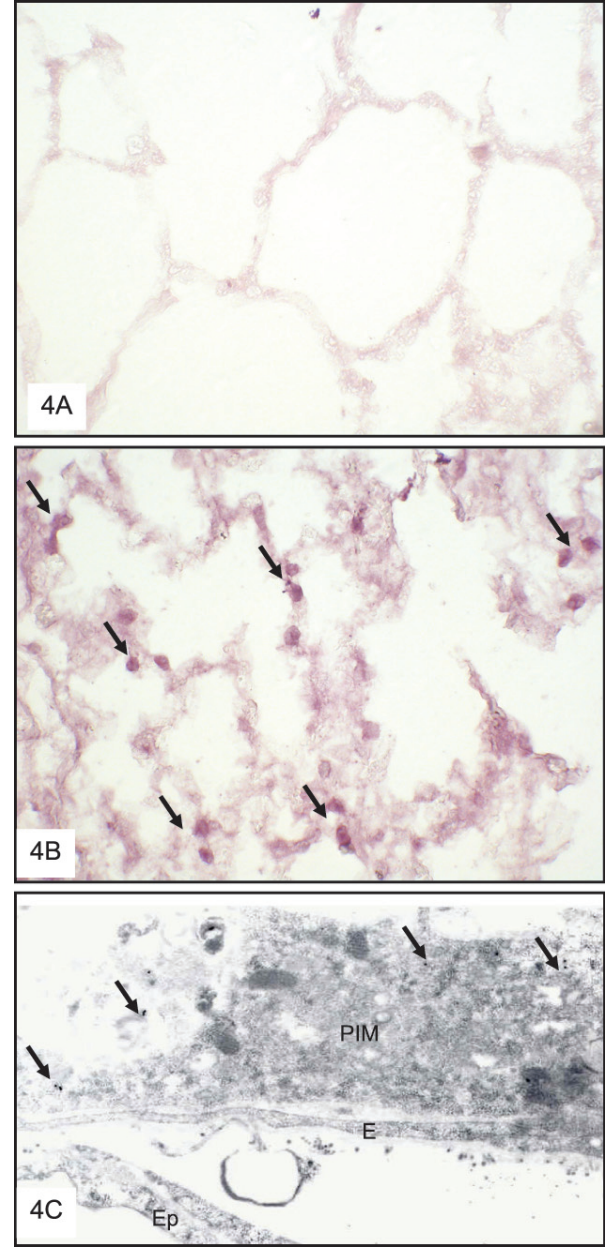

Figure 4. IL-1 $\beta$ immunocytochemistry: Lung section from a control horse (A) shows a faint background staining in the alveolar septa and an occasional cell. Intense staining is evident in the alveolar septa and discrete septal cells (arrows) in lung sections from saline + LPS-treated horses (B). An immuno-gold electron micrograph (C) shows gold particles (arrows) indicative of labeling for IL-1 $\beta$ in a PIM from a saline + LPStreated horse. E: endothelium; Ep: epithelium. A, B: $\times 400$; $\mathrm{C}: \times 37500$ (for a colour version of this figure see www.edpsciences.org/vetres).

were in agreement with evidence from other species. Our experiments did not address the mechanisms of uptake of LPS

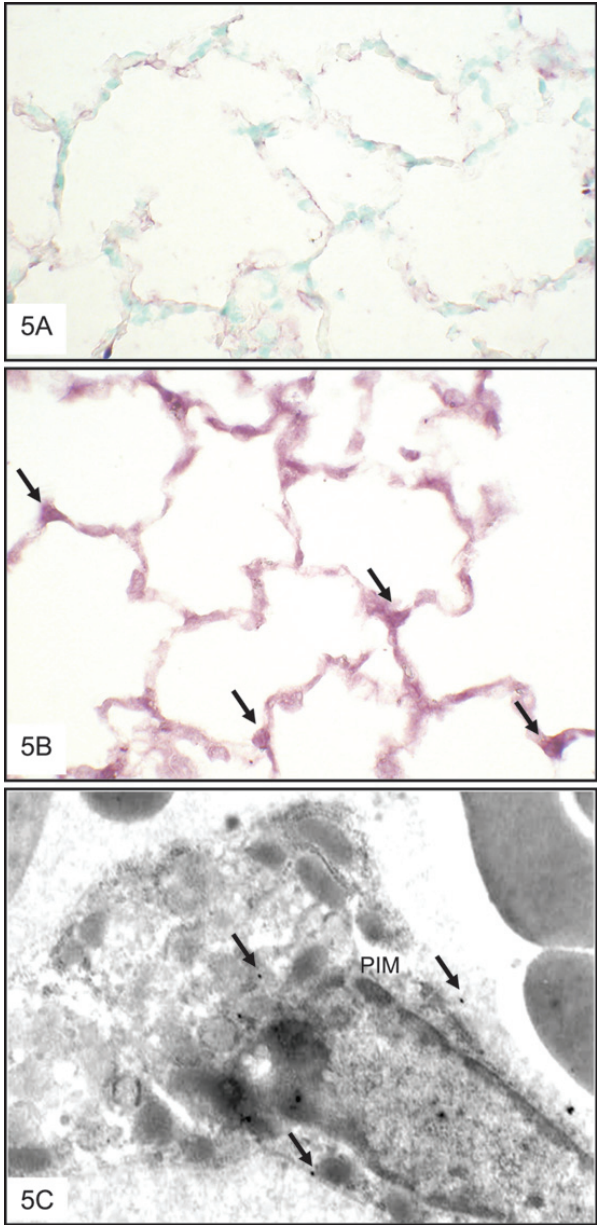

Figure 5. TNF- $\alpha$ immunocytochemistry: Lung section from a control horse (A) lacks staining for TNF- $\alpha$ while that from a saline + LPS treated horse (B) shows intense staining in many large septal cells (arrows). An electron micrograph (C) depicts gold particles (arrows) indicative of TNF- $\alpha$ staining in a PIM in a lung section from saline + LPS treated horse. A, B: $\times 400$; C: $\times 37500$ (for a colour version of this figure see www.edpsciences.org/vetres).

by equine PIMs. It is well established that macrophages contain CD14 and TLR4 which are critical for endocytosis of LPS, induction of cell signaling and transcription of pro-inflammatory genes $[1,2,12]$. Our 

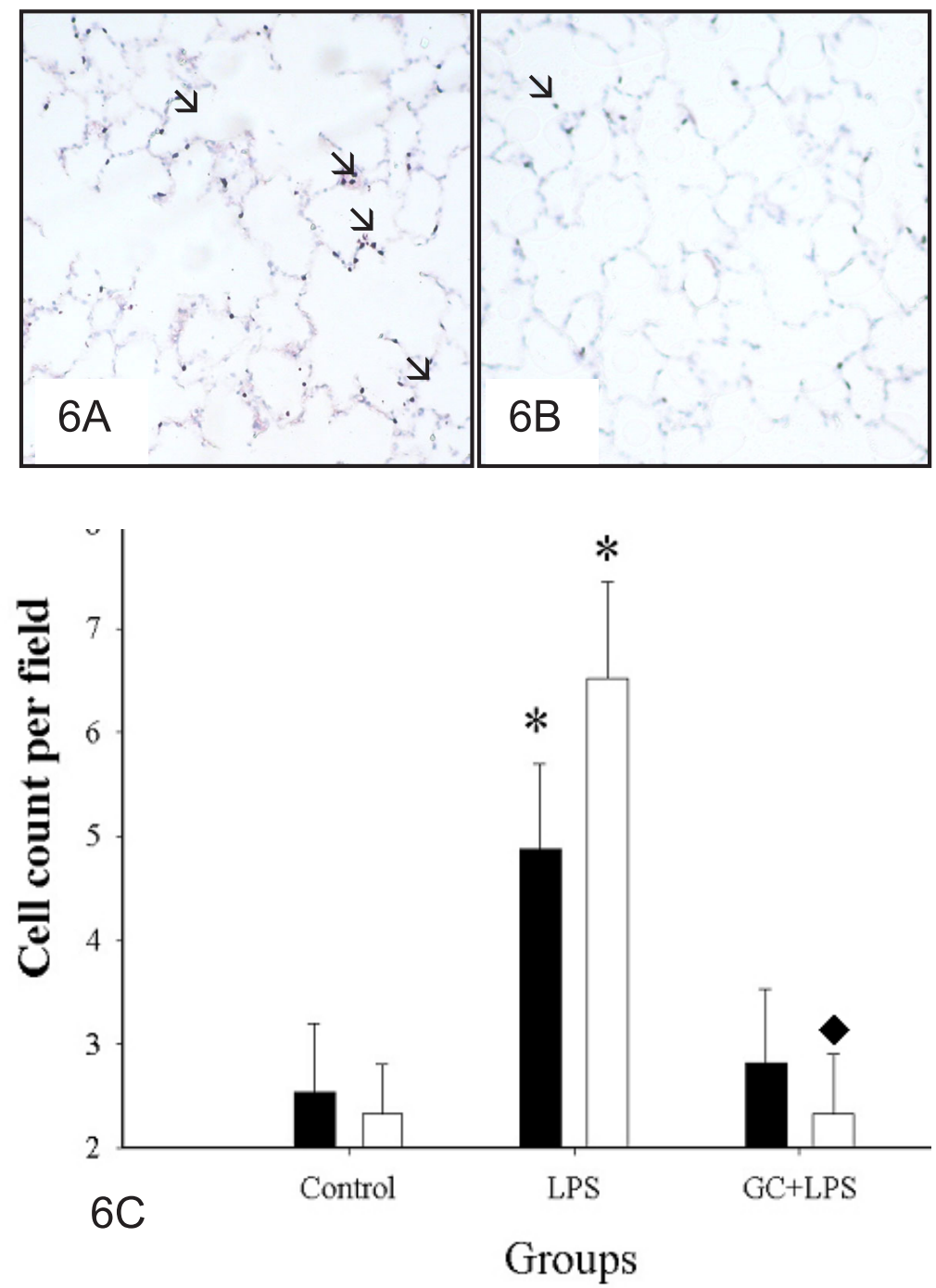

Figure 6. MAC387 immunohistochemistry and numerical counts for TNF- $\alpha$ and IL-1 $\beta$ : Lung section from a normal horse (6A) shows many MAC-387 positive septal cells (arrows) compared to the section (6B) from a GC-treated horse. Numerical counts were made in 20 fields under oil immersion ( $\times 100)$ from one section from each tissue block (7 blocks/lung; 14 blocks/horse). Each field comprised $0.025 \mathrm{~mm}^{2}$ of tissue area, which resulted in $0.50 \mathrm{~mm}^{2} / \mathrm{section}$ and $7.0 \mathrm{~mm}^{2} /$ horse. The numbers of TNF- $\alpha$ (solid bars) and IL-1 $\beta$ (open bars) were significantly increased $(* P<0.05)$ in both the LPS-treated groups compared to the controls (6C). GC + LPS horses, compared to the LPStreated horses, showed significant reduction $(\diamond P<0.05)$ in cells positive for IL-1 $\beta$ (open bars) but not for TNF- $\alpha$ (solid bars). Original magnification: $\times 10$ (for a colour version of this figure see www.edpsciences.org/vetres). 
preliminary data reveal immunohistochemical staining for TLR4 in equine PIMs (B. Singh, unpublished data). Therefore, equine PIMs may have the molecular machinery to engage and endocytose LPS as a critical step in their activation, and we provide the first evidence of in vivo LPS uptake by equine PIMs.

Till date, there are no protocols to isolate PIMs for in vitro studies to assess their cell biology. Therefore, we recently developed a method to selectively reduce the numbers of equine PIMs, without affecting alveolar or liver macrophages, with a single injection of GC [23]. GC is selectively phagocytosed by PIMs due to the first pass phenomenon following its infusion in the jugular vein. GC activates apoptosis in equine PIMs and induces significant depletion within $48 \mathrm{~h}$ of the treatment [23]. In the current experiments, the horses treated with GC showed reduced numbers of PIMs in their lungs. Therefore, we have now used this model to define pathophysiological contributions of PIMs by comparing LPSinduced lung responses in horses with intact or depleted PIMs. The horses treated with the $50 \mathrm{ng} / \mathrm{kg}$ dose of LPS showed pulmonary arterial hypertension. These observations were in agreement with data previously reported by Longworth et al. [17]. PIM depletion of LPS-treated horses inhibited increases in mean, but not in diastolic or systolic pulmonary arterial pressures compared to the normal saline-treated horses. The reasons for this dissociation in response to diastolic or systolic, compared to the mean, pulmonary arterial pressures are not known. However, it is possible that a larger sample size may have resulted in a significant response in diastolic and systolic pressures. Although the vasoactive mediators secreted by the equine PIMs remain unknown, it is possible that as with porcine PIMs, these cells may produce arachidonic acid metabolites following the uptake of the LPS $[7,8]$. Therefore, PIM depletion will eliminate a vascular source of pro-inflammatory vasoactive substances to reduce LPSinduced pulmonary arterial hypertension.
We examined equine PIMs for TNF- $\alpha$ and IL- $1 \beta$ with light and electron microscopic immunocytochemistry and provide the first localization of these cytokines in the PIMs and alveolar septal cells of the LPS-treated horses. Macrophages as well as endothelial and epithelial cells are activated following interactions with the LPS and produce pro-inflammatory cytokines $[1,10]$. TNF- $\alpha$ and IL- $1 \beta$, secreted by macrophages, are considered critical for the initiation of the endotoxin-induced inflammatory cascade $[13,22]$. Because IL- $1 \beta$ and TNF- $\alpha$ induce the expression of adhesion molecules and migration of neutrophils in inflamed organs $[14,20]$, we counted the number of cells positive for these cytokines as an indicator for LPS-induced inflammation. The LPS-treatment of control horses increased the number of cells positive for TNF- $\alpha$ or IL- $1 \beta$ compared to the control saline-treated horses. TNF- $\alpha$ and IL- $1 \beta$ staining was also observed in the septal cells of LPS-treated horses. This was in agreement with the previous data that alveolar epithelial and microvascular endothelial cells secrete TNF- $\alpha$ and IL-1 $\beta$ [10]. However, PIM-depleted LPS-treated horses showed a significant decline in the numbers of septal cells positive for IL- $\beta$, but not TNF- $\alpha$, compared to the normal LPStreated horses. It is possible that reduced numbers of IL- $1 \beta$ positive cells in GC and LPS-treated horses may be a combined effect of the direct elimination of PIMs and down-stream effects of their elimination on other cells that contain IL-1 $\beta$. Interestingly, IL- $1 \beta$, compared to TNF- $\alpha$, plays a more dominant role in the early phase of LPSinduced lung inflammation [10]. There is evidence that mice deficient in the production of mature IL- $1 \beta$ are resistant to endotoxic shock [15]. However, in vivo administration of an antibody against TNF- $\alpha$ did not cause any therapeutic effect in endotoxemic horses [6]. In this context, the inhibition of LPS-induced increase in IL- $1 \beta$ cells in PIM-depleted horses may critically modulate the early phase of endotoxin-induced pulmonary pathophysiology. 
Finally, for the following reasons, we caution against wide-ranging extrapolation of the data reported in this paper. First, we focused only on the very early pulmonary events in the LPS-treated horses. Second, although LPS-induced pulmonary disease is a multifactorial event, our observations were limited to the examination of a single cell and only two of the relevant cytokines. Third, the role of novel molecules such as TLR4 in endotoxin-induced lung inflammation in horses is yet to be determined. Despite these limitations, our data are the first direct evidence that PIMs contain proinflammatory cytokines and a reduction in their numbers potentially inhibits the LPSinduced pulmonary vascular and inflammatory events. These data are similar to recently reported evidence that PIM depletion inhibits Mannheimia hemolytica-induced lung inflammation in calves [26]. Therefore, PIMs may represent a strikingly new therapeutic target to modulate lung inflammation in horses and ruminants once more studies are completed to clarify their specific contributions.

\section{ACKNOWLEDGEMENTS}

This study was supported by a grant from Alberta Agriculture Research Institute to Dr Baljit Singh. Dr Parbhakar was a recipient of a Graduate Student Fellowship from the Interprovincial Fund of the Western College of Veterinary Medicine. We thank Ms. Sarah Caldwell for help with immuno-electron microscopy.

\section{REFERENCES}

[1] Aderem A., Role of Toll-like receptors in inflammatory response in macrophages, Crit. Care Med. 29 (2001) S16-S18.

[2] Aderem A., Underhill D.M., Mechanisms of phagocytosis in macrophages, Annu. Rev. Immunol. 17 (1999) 593-623.

[3] Atwal O.S., Saldanha K.A., Erythrophagocytosis in alveolar capillaries of goat lung: Ultrastructural properties of blood monocytes, Acta Anat. 124 (1985) 245-254.
[4] Atwal O.S., Singh B., Staempfli H., Minhas K.J., Presence of pulmonary intravascular macrophages in the equine lung: Some structuro-functional properties, Anat. Rec. 234 (1992) 530-540.

[5] Atwal O.S., McDonell W., Staempfli H., Singh B., Minhas K.J., Evidence that halothane anaesthesia induces intracellular translocation of surface coat and Golgi response in equine pulmonary intravascular macrophages, J. Submicrosc. Cytol. Pathol. 26 (1994) 369386.

[6] Barton M.H., Bruce E.H., Moore J.N., Norton N., Anders B., Morris D.D., Effect of tumor necrosis factor antibody given to horses during early experimentally induced endotoxemia, Am. J. Vet. Res. 59 (1998) 792-797.

[7] Chitko-McKown C.G., Blecha F., Pulmonary intravascular macrophages: a review of immune properties and functions, Ann. Rech. Vet. 23 (1992) 201-214.

[8] Chitko-McKown C.G., Chapes S.K., Brown R.E., Phillips R.M., McKown R.D., Blecha F., Porcine alveolar and pulmonary intravascular macrophages: Comparison of immune functions, J. Leukoc. Biol. 50 (1991) 364-372.

[9] Decamp M.M., Warner A.E., Molina R.M., Brain J.D., Hepatic versus pulmonary uptake of particles injected into the portal circulation in sheep, Am. Rev. Resp. Dis. 146 (1992) 224-231.

[10] Dinarello C.A., The interleukin-1 family: 10 years of discovery, FASEB J. 8 (1994) 1314 1325 .

[11] Fein A.M., Calalang-Colucci M.G., Acute lung injury and acute respiratory distress syndrome in sepsis and septic shock, Crit. Care Clin. 16 (2000) 289-317.

[12] Kang Y.-H., Lee C.-H., Monroy R.L., Dwivedi R.S., Odeyale C., Newball H.H., Uptake, distribution and fate of bacterial lipopolysaccharides in monocytes and macrophages: An ultrastructural and function correlation, Electron Microsc. Rev. 5 (1992) 381-419.

[13] Koay M.A., Gao X., Washington M.K., Parman K.S., Sadikot R.T., Blackwell T.S., Christman J.W., Macrophages are necessary for maximal nuclear factor-kappa B activation in response to endotoxin, Am. J. Respir. Cell Mol. Biol. 26 (2002) 572-578.

[14] Kubes P., Jutila M., Payne D., Therapeutic potential of inhibiting leukocyte rolling in ischemia/reperfusion, J. Clin. Invest. 95 (1999) 2510-2519. 
[15] Li P., Allen H., Banerjee S., Franklin S., Herzog L., Johnston C., McDowell J., Paskind M., Rodman L., Salfeld J., Towne E., Tracey D., Wardwell S., Wei F.-Y., Wong W., Kamen R., Seshadri T., Mice deficient in IL-1b-converting enzyme are defective in production of mature IL-1b are resistant to endotoxic shock, Cell 80 (1995) 401-411.

[16] Longworth K.E., Jarvis K.A., Tyler W.S., Steffey E.P., Staub N.C., Pulmonary intravascular macrophages in horses and ponies, Am. J. Vet. Res. 55 (1994) 382-388.

[17] Longworth K.E., Smith B.L., Staub N.C., Steffey E.P., Serikov V.B., Use of detergent to prevent initial responses to endotoxin in horses, Am. J. Vet. Res. 57 (1996) 10631066.

[18] Marsik C., Mayr F., Cardona F., Derhaschnig U., Wagner O.F., Jilma B., Endotoxemia modulates Toll-like receptors on leucocytes in humans, Br. J. Haematol. 121 (2003) 653656.

[19] Miyamoto K., Schultz E., Heath T., Mitchell M.D., Albertine K.H., Staub N.C., Pulmonary intravascular macrophages and hemodynamic effects of liposomes in sheep, J. Appl. Physiol. 64 (1988) 1143-1152.

[20] Mizgerd J.P., Molecular mechanisms of neutrophil recruitment elicited by bacteria in the lungs, Semin. Immunol. 14 (2002) 123-132.

[21] Morris D.D., Endotoxemia in horses: A review of cellular and humoral mediators involved in its pathogenesis, J. Vet. Int. Med. 5 (1991) 167-181.

[22] Oshikawa K., Sugiyama Y., Gene expression of Toll-like receptors and associated molecules induced by inflammatory stimuli in the primary alveolar macrophage, Biochem. Biophys. Res. Commun. 305 (2003) 649-655.

[23] Parbhakar O., Duke T., Townsend H.G., Singh B., Immunophenotypic characterization and depletion of pulmonary intravascular macrophages of horses, Vet. Res. 35 (2004) 39-52.

[24] Singh B., Atwal O.S., Ultrastructural and immunocytochemical study of the pulmonary intravascular macrophages of Escherichia coli lipopolysaccharide-treated sheep, Anat. Rec. 247 (1997) 214-224.
[25] Singh B., Minhas K.J., Atwal O.S., Ultracytochemical study of multiple dose effect of monastral blue uptake by equine pulmonary intravascular macrophages (PIMs), J. Submicrosc. Cytol. Pathol. 26 (1994) 235-243.

[26] Singh B., Pearce J.W., Gamage L.N.A., Janardhan K., Caldwell S., Depletion of pulmonary intravascular macrophages inhibits acute lung inflammation, Am. J. Physiol. 286 (2004) L363-L372.

[27] Sone Y., Nicolaysen A., Staub N.C., Effect of particles on sheep lung hemodynamics parallels depletion and recovery of intravascular macrophages, J. Appl. Physiol. 83 (1997) 1499-1507.

[28] Sone Y., Serikov V.B., Staub N.C., Intravascular macrophage depletion attenuates endotoxin lung injury in anesthetized sheep, J. Appl. Physiol. 87 (1999) 1354-1359.

[29] Staub N.C., Pulmonary intravascular macrophages, Ann. Rev. Physiol. 56 (1994) 47-67.

[30] Staub N.C., Longworth K.E., Serikov V.B., Jerome E.H., Elsasser T.H., Detergent inhibits $70-90 \%$ of responses to intravenous endotoxin in awake sheep, J. Appl. Physiol. 90 (2001) 1788-1797.

[31] Traub-Dargatz J.L., Salman M.D., Voss J.L., Medical problems of adult horses, as ranked by equine practitioners, JAVMA 198 (1991) 1745-1747.

[32] Warner A.E., Pulmonary intravascular macrophages: Role in acute lung injury, Clin. Chest Med. 17 (1996) 125-135.

[33] Warner A.E., Barry B.A., Brain J.D., Pulmonary intravascular macrophages in sheep: Morphology and function of a novel constituent of the mononuclear phagocyte system, Lab. Invest. 55 (1986) 276-288.

[34] Warner A.E., Decamp M.M., Molina R.M., Brain J.D., Pulmonary removal of circulating endotoxin results in acute lung injury in sheep, Lab. Invest. 59 (1988) 219-230.

[35] Xavier A.M., Isowa N., Cai L., Dziak E., Opas M., McRitchie D.I., Slutsky A.S., Keshavjee S.H., Liu M., Tumor necrosis factor-alpha mediates lipopolysaccharide-induced macrophage inflammatory protein-2 release from alveolar epithelial cells. Autoregulation in host defense, Am. J. Respir. Cell Mol. Biol. 21 (1999) 510-520. 